\title{
Hybridation linguistique et identité culturelle au Sénégal
}

\author{
Dr. Ibrahima Sarr, \\ Université Gaston Berger de Saint-Louis \\ Laboratoire Recherches Sociolinguistiques et Didactiques, Senegal
}

Doi:10.19044/esj.2020.v16n23p102 URL:http://dx.doi.org/10.19044/esj.2020.v16n23p102

\section{Résumé}

La question de la vie des langues dans les contextes multilingues est un sujet d'intérêt interdisciplinaire (histoire, politique, sociolinguistique, linguistique etc.) Dans ce domaine, les phénomènes d'hybridations linguistiques ont focalisé ces dernières années des recherches multisectorielles pour des raisons liées à leurs enjeux identitaires, leurs implications linguistiques entre autres. Cet article s'applique au contexte sénégalais caractérisé par un multilinguisme actif pour interroger les implications identitaires des hybrides linguistiques dans le contexte de lutte pour la valorisation des langues nationales restées sous le joug du français, langue officielle. Il offre également une analyse des positionnements épilinguistiques générés par ces hybrides. L'article aborde la question sous deux angles: un angle diachronique pour saisir les évolutions du paysage sociolinguistique sénégalais et les dynamiques qui ont favorisé le processus d'hybridation, et un angle synchronique pour donner une vue d'ensemble du phénomène et sa perception par les locuteurs. Cet article s'appuie sur un travail de terrain effectué auprès de la population estudiantine de Saint-Louis et des media de la localité pour le rôle qu'ils jouent dans la création lexicale et la diffusions des parlures nationales. L'analyse des données recueillies montre clairement que l'hybridation linguistique est bien en cours dans le paysage linguistique sénégalais et qu'elle tire sa substance des langues étrangères et des langues locales. C'est un phénomène qui se généraliser d'autant plus que les identités en constructions en font un élément fondateur.

Mots-clés : Hybride linguistique, identité, parlures nationales, Sénégal 


\title{
Linguistic Hybridization and Cultural Identity in Senegal
}

\author{
Dr. Ibrahima Sarr, \\ Université Gaston Berger de Saint-Louis \\ Laboratoire Recherches Sociolinguistiques et Didactiques, Senegal
}

\begin{abstract}
The issue of languages in multilingual contexts is a subject of interdisciplinary interest (history, politics, sociolinguistics, linguistics, etc.) In this field, phenomena of linguistic hybridization have focused in recent years on multisectoral research for reasons related to their identity issues, their linguistic implications among others. This article applies to the Senegalese context characterized by active multilingualism to question the identity implications of linguistic hybrids in the context of the struggle for the empowerment of national languages that have remained under the yoke of French, the official language. It also offers an analysis of the linguistic attitudes generated by these hybrids. The article approaches the question from two angles: a diachronic angle to grasp the evolutions of the Senegalese sociolinguistic landscape and the dynamics that favored the process of hybridization, and a synchronic angle to give an overview of the phenomenon and its perception by speakers. This article is based on a fieldwork carried out with the student population of Saint-Louis and local media for the role they play in lexical creation and dissemination of national languages. The analysis of the data collected clearly shows that linguistic hybridization is in progress within the Senegalese sociolinguistic environment. Besides, it draws from the contact between foreign and local languages. That phenomenon is expanding all the more as it constitute the bedrock of the construction of identity.
\end{abstract}

Keywords: Hybrids, identity, national languages, Senegal

\section{Introduction}

Les pratiques linguistiques en contexte diglossique ont polarisé l'attention de beaucoup de chercheurs en sociolinguistique et au-delà. Son intérêt est d'autant plus grand sur le terrain africain qui est un creuset linguistique ou il est estime qu'environ un tiers des langues du monde sont 
parlées avec des chiffres par pays pouvant atteindre les quatre cent langues5. Cette pluralité des langues, la cohabitation des locuteurs due aux migrations historiques et récentes, la croisée des langues d'Afrique et d'ailleurs et d'autres facteurs encore intensifient les contacts de langues et complexifient les pratiques langagières des locuteurs. Ces pratiques, quelque soient les réalités qui les motivent, tendent souvent vers un phénomène d'hybridation pouvant prendre plusieurs formes selon les contextes. Les locuteurs, de façon consciente ou pas, prennent ainsi activement part à la construction des hybrides linguistiques dans leurs différentes formes d'expression. Cependant, nous ne pourrions épuiser notre sujet si nous n'abordions pas la question de l'identité culturelle des locuteurs car comme le souligne Kerry Mullan, « La question de l'identité culturelle n'est jamais loin de toute langue hybride [...] ».6 Cela est d'autant plus important que notre travail s'inscrit dans un contexte de lutte pour l'émergence des parlures nationales au Sénégal, et donc de résistance à l'hégémonie de la langue officielle. Pourtant, l'on intègre progressivement l'idée que le Sénégalais standard n'est pas celui-là qui a su se préserver de toute influence étrangère mais plutôt celui qui a forgé son identité en intégrant la mixité née des croisements de la civilisation africaine, européenne et arabo-islamique. C'est ce que soutiennent en tout cas Issa Diallo et al. Lorsqu'ils écrivent que «La réalité des francophones de l'Afrique subsaharienne est caractérisée par le multilinguisme fonctionnel ou les langues de communication de masse ne sont pas toujours celle de l'environnement scolaire, dominé en francophonie par le français $»^{7}$. Cela se vérifie, en effet, pour le Sénégal la politique linguistique de l'Etat reconnait le français comme langue officielle et médium d'instruction alors que le wolof (parlée par plus de $80 \%$ de la population) demeure la langue de communication de la grande masse.

Autant dire donc que dans cette mondialité, la défense de la diversité s'érige partout en norme, ce qui passe en quelque sorte par la promotion de l'hybride.

\section{Approche conceptuelle \\ 1. Hybridation linguistique}

L'hybride est, de manière générale, le produit d'un croisement. Son sens peut varier selon le contexte d'utilisation comme en botanique, en zoologie, en mécanique etc. Dans le champ de la sociolinguistique, l'hybride

\footnotetext{
${ }^{5}$ Le Nigeria compte à lui seul environ 527 langues, le Cameroun en a plus de 250.

${ }^{6}$ Kerry Mullan, « Henri Boyer (éd.), Hybrides linguistiques. » p. 388.

7 Issa Diallo et al. « Bi-grammaire fulfulde-français » (version de travail) in Développement de l'enseignement des langues africaines : le français en contexte multilingue. OIF (sans date) http://www.universitepopulairemeroeafrica.org/IMG/pdf/Bigrammaire_fulfude_francais.pdf
} 
linguistique renvoie à une langue mixte où les emplois des locuteurs vacillent entre les frontières de plusieurs langues. L'hybride linguistique est cependant parfois vu comme un continuum dialectal, c'est-à-dire comme une forme de variation dont les caractéristiques linguistiques sont un lexique, une grammaire et des propriétés phonologiques polyhybrides. Vu sous cette angle, l'hybridation serait ainsi le processus par lequel les locuteurs d'une langue utilisent et finissent par intégrer dans leurs pratiques langagières des formes transcodiques.

\section{Identité culturelle}

Le processus d'hybridation linguistique va de pair avec le remoulage de l'identité culturelle ${ }^{8}$ des locuteurs dans la mesure où la langue remplit une fonction identitaire et que la langue que nous parlons et la façon dont nous la parlons disent notre personnalité, notre niveau d'instruction, notre origine géographique, notre statut socioprofessionnel. Il faut reconnaitre que dans notre monde globalisé, le thème de recherche le plus en vogue, celui qui détermine nos positionnements et nos actions dans nos rapports à notre environnement, c'est bien l'identité. Même si elle est difficile à saisir du fait de sa nature plurielle, variable et dynamique, l'analyse du discours épilinguistique des locuteurs permettra d'en saisir certains aspects importants.

\section{Parlures}

Comme pour le concept d' "hybridation», celui de «parlure » est caractérisé par sa variabilité sémantique. Dans son ancienne acception, une parlure est une langue. En sociolinguistique, elle renvoie à une manière de parler caractéristique d'un groupe social donné ; autrement dit l'ensemble des moyens d'expression d'un groupe social. En cela, la parlure est assimilable à un sociolecte qui est une variation de la langue selon les usagers (François Gadet, 2003). Dans le présent travail, le terme « parlure » est considéré avec cette deuxième acception car les pratiques que nous analysons sont principalement le fait de groupes sociaux identifiables, même si ses frontières s'étendent de jour en jour à tous les locuteurs, quelle que soit la variable sociologique considérée.

\section{Approche méthodologique}

Ce travail se base d'abord sur une recherche documentaire ensuite sur des données tirées de communications médiées et d'autres issues d'un travail de terrain conduit auprès des étudiants de l'Université Gaston Berger de SaintLouis et des populations de la ville de Saint-Louis. Il s'agit en quelque sorte

${ }^{8}$ Le processus grâce auquel un groupe d'individus prend conscience de sa manière partiellement commune de comprendre l'univers, d'agir sur lui et de communiquer ses idées et ses modèles d'action. 
de couvrir différents profiles pour mieux apprécier la nature sociétale des faits que nous cherchons à appréhender.

La recherche documentaire a permis de parcourir des travaux allant des mémoires de recherche des étudiants aux thèses de doctorats en passant par les articles scientifiques et les ouvrages consacrés à la thématique des hybrides linguistiques et leurs implications identitaires dans le contexte sociolinguistique sénégalais. Il s'est agi de jeter un regard sur la question de la construction/reconstruction des identités à travers les jeux linguistiques mais également de passer en revue les travaux de sociolinguistiques consacrés au paysage sociolinguistique sénégalais qui s'est avéré très propice aux pratiques d'hybridation. Les politiques linguistiques et la structuration qu'elles ont établies dans la vie des langues ont été également édifiantes dans l'orientation des pratiques langagières des locuteurs.

Pour ce qui concerne le travail de terrain proprement dit, il a d'abord consisté en une pré-enquête auprès des cibles pour avoir un aperçu de la situation; ensuite de l'enquête elle-même qui s'est faite par observation et par entretien. C'est ce qui a permis de collecter des données sur les pratiques langagières et sur les positionnements épilinguistiques des locuteurs et la perception qu'ils ont de leur identité. Les sujets, qui sont des acteurs de notre environnent médiatique, pédagogique et/ou sociale, ont été observés dans différents contextes pour saisir une éventuelle variabilité dans leurs pratiques langagières selon les contextes dans lesquels ils interagissent. Les données recueillies par observation et par entretien semi-directif ont ensuite été soumises à une analyse sociolinguistique pour cerner les contextes d'apparition des hybrides linguistiques, leurs caractéristiques linguistiques et les attitudes les locuteurs à travers la teneur de leur discours épilinguistique.

\section{Genèse et statut des parlures nationales au Sénégal}

Les langues nationales du Sénégal ont une histoire semblable à celle des langues autochtones des pays qui ont subi la domination coloniale française. Elles sont, comme leurs locuteurs, les victimes d'une politique d'assimilation qui les a maintenues sous le joug du français dont elles peinent encore à se libérer malgré les efforts d'émancipation et la volonté politique de l'Etat, des activistes et des spécialistes. Le statut que ces langues ont acquis aujourd'hui est ainsi le résultat d'une longue lutte. L'usage des langues locales, en plus de leur valeur patrimoniale, a souvent été un moyen de résistance à l'acculturation, surtout pour ceux qui prônent la pureté linguistique. C'est pour cela que la question de leur écriture s'est posée très tôt à partir du XI ${ }^{\text {ème }}$ siècle alors qu'elles étaient encore sous le joug de l'impérialisme arabo-musulman. C'est là que l'alphabet Ajami s'est développé avant que la conférence de Bamako (1966) et celle de Niamey 
(1978) viennent marquer la préférence de ce qui deviendra plus tard l'alphabet africain de référence (AAR).

Retenons aussi que c'est en 1978 que le Sénégal validera les normes de codification de six langues locales sur une trentaine auxquelles il confère le statut de langue nationale. Il s'agissait alors du wolof, du sereer, du pulaar, du manding, du joola et du saraxolle ${ }^{9}$ qui étaient démographiquement les langues locales dominantes. Notons que les mesures d'aménagement ont été plus timides car le constat est que ces nouveaux statuts n'ont pas eu les garanties qui devaient permettre à ces langues de jouer des rôles majeurs dans la vie des locuteurs du fait que la distribution fonctionnelle était totalement en faveur du français, langue officielle. Les choses resteront ainsi jusqu'en 2001 avec les amendements de la constitution sénégalaise qui assouplissent les critères de promotion des langues locales et étendent le statut de langue nationale à toutes les langues codifiées. Pourtant jusque-là la législation linguistique ne leur attribue pas directement des fonctions hormis l'objectif exprimé d'en faire des langues de culture comme cela est écrit dans le rapport de présentation des décrets sur l'orthographe et la séparation des mots dans les langues nationales du Sénégal :

L'objectif de faire des langues nationales sénégalaises des langues de culture et, par la même occasion, de donner plus de moyens et d'efficacité à l'éducation, à la modernité et aux efforts de développement, exige que ces langues soient écrites, introduites dans le système éducatif et utilisées dans la vie officielle et publique ${ }^{10}$.

Même si l'intention affichée dans cette constitution est louable, nous pouvons quand même en déceler quelques manquements: il s'agit premièrement du fait que la constitution ne définit pas ce qu'est une langue nationale ; puis du fait qu'elle ne leur attribue aucun rôle et enfin du fait qu'elle n'en limite pas le nombre.

Par ailleurs, même si le Français demeure la langue officielle du Sénégal et qu'elle a régné en maitre absolu pendant une longue période dans toutes les sphères de communication officielle, bénéficiant de l'attitude très positive des locuteurs du fait qu'elle constituait une clé déterminante à l'insertion professionnelle, force est de reconnaitre avec Ndao et Kébé ${ }^{11}$ que

\footnotetext{
${ }^{9}$ Les noms des langues nationales sont ici transcrits conformément aux directives des décrets qui les ont promus. Le dédoublement de la voyelle marque phonologiquement la longueur vocalique alors que celui de la consonne marque la gémination.

${ }^{10}$ Journal Officiel n ${ }^{\circ} 6280$ du samedi 27 mai 2006 http://www.j.o.gouv.sn/spip.php?article4800.

${ }^{11}$ Papa Aliou Ndao \& Abou Bakry Kébé, 2010, « Langues et médias au Sénégal : une expérience de normalisation langagière par les journalistes des radios privées. Enjeux et
} 
ces dernières années ont été surtout favorables à l'irruption des langues nationales du Sénégal dans des sphères jadis dévolues au Français. Ce revirement a été rendu possible grâce à une série d'actes de haute portée politique comme les amendements de la constitution de 2001, la décision d'Abdoulaye Wade de septembre 2001 d'alphabétiser tous les fonctionnaires de l'Etat en langue nationale, l'usage quasi-systématique des langues nationales au côté du français dans les communications de l'Etat, l'introduction des langues nationales dans le système éducatif à partir des classes pilotes $^{12}$, la mise à l'échelle de l'enseignement bilingue depuis 2017 , entre autres. Il faut constater qu'un tel changement dans les pratiques linguistiques des locuteurs et dans la distribution fonctionnelle des langues ${ }^{13}$ découle quelque part d'une évolution dans la conception que les locuteurs se font de leur identité linguistique qui détermine donc leurs rapports à l'autre langue, le «they code » (Gumperz, 1976). C'est aussi cette redéfinition des identités collectives intégrant divers apports linguistiques et culturels qui motive et consolide les hybrides linguistiques dans le contexte sénégalais.

\section{Contextes d'apparition des hybrides linguistiques au Sénégal}

Le Sénégal est un creuset linguistique où on décompte une trentaine de langues. Si en remontant à l'histoire du peuplement du pays, on arrive à identifier et à localiser avec exactitude les différents foyers de peuplement dédiés aux différents groupes linguistiques, aujourd'hui, les migrations ont eu comme conséquences linguistiques l'éclatement des aires géolinguistiques, entrainant une diversification des parlers et des variétés linguistiques en usage à travers les différentes sphères d'expression de ces langues. Ce contexte de décloisonnement favorise le contact de langues et de locuteurs qui fait apparaitre crée une situation diglossique. Ce multilinguisme appelle l'interventionnisme de l'Etat qui, dans sa politique linguistique procède à une distribution fonctionnelle où le français reçoit la part du lion provoquant un sentiment d'insécurité chez les locuteurs des langues autochtones. Cependant, les garanties que confère l'usage du français font qu'il est généralement

limites », dans Ndao P. A. \& Kébé A. B., (dirs.) Nouveaux médias et dynamiques des langues dans l'espace francophone, Glottopol $\mathrm{n}^{\circ} 14$, en ligne sur http://www.univrouen.fr/dyalang/glottopol/, pp. 17-36, (accès le 21.10.2018).

${ }^{12}$ L'essai qui a abouti à la mise à l'échelle de l'enseignement bilingue a été conduit par l'ONG Association for Research and Development (ARED)

${ }^{13}$ Même si la législation linguistique dans ce domaine n'a pas autant changé du fait que la Constitution accorde toujours le statut de langue officielle au français et reste plutôt muette sur les rôles des langues nationales, dans les faits, les guerre des langues tourne nettement en faveur des langues nationales aussi bien dans les attitudes que dans les pratiques linguistiques des locuteurs, y compris à tous les niveaux de la vie étatique et dans les média (surtout la presse audiovisuelle). 
accepté et même enrichi avec les apports multiples des autres langues de l'environnement consolidant ainsi la diversité linguistique comme richesse. Dans les pratiques langagières, le contact de langues et les attitudes positives des locuteurs donnent lieu à un transcodisme où les locuteurs ne tarissent pas d'initiatives. Les formes résultantes sont ainsi soumises à la variabilité selon les combinaisons linguistiques des locuteurs, les situations de communication et les effets de sens souhaités. Les positionnements épilinguistiques par rapport à ces hybrides linguistiques varient selon qu'on se situe du côté de la défense des langues nationales où qu'on reste neutre en laissant libre court à la glottophagie. Les locuteurs peuvent ainsi se retrouver dans une situation de semilinguisme en développant une culture de l' « entre deux ». Ils ne seront pas de parfaits bilingues car la pratique des formes hybrides provoque à la longue une perte partielle des compétences antérieurement établies chez les praticiens.

\section{Les particularités linguistiques des hybrides linguistiques au Sénégal}

Pour analyser les hybrides linguistiques nous nous baserons sur des extraits de notre corpus. L'analyse concerne divers angles (linguistique, sociolinguistique, politique) sans qu'ils ne soient nécessairement exclusifs.

\section{Extrait corpus 1 : les hybrides dans les média ${ }^{14}$}

Extrait 1 corpus 1 : Il s'agit d'une Conversation téléphonique entre Son-J et N'douba, deux acteurs du téléfilm Tund-wund ${ }^{15}$ que Sarr présente comme :

un film où la question linguistique se pose dès

l'entame. Le titre wolof est une métaphore qui traduit une vie d'individualisme où chacun n'hésite pas trahir l'autre pour s'emparer d'un butin. Mais si le titre est wolof, le synopsis est présenté en français ainsi que le carton ce qui l'installe confortablement dans un registre de bilinguisme et de mélange de langues. Le cadre physique du tournage passe du bureau à la maison en passant par la rue et couvre une diversité de langues et de pratiques linguistiques qui portent les

\footnotetext{
${ }^{14}$ Ce passage est extrait de l'épisode 1 du téléfilm Sénégalais Tund-wund

15 « Tundu Wundu » est le titre d'un feuilleton sénégalais qui dépeint le quotidien de la vie urbaine, le monde impitoyable et inhumain de la politique miné par la corruption et le gangstérisme et les conditions difficiles des basses couches laissées à elles-mêmes, sans défense et devant se battre pour survivre dans cette jungle. Il part d'une histoire d'amour entre le jeune Badara et Myriam qui tourne vite au vinaigre lorsque, accusé d'avoir assassiné Draman, l'ex époux de Myriam, Badara est incarcéré. Ayant obtenu une liberté provisoire, il se voit rejeté par son père qui le présume coupable des faits qui lui sont imputés.
} 
différences de groupes, de statuts socioprofessionnels et de milieux ${ }^{16}$.

Son-J : Allô !

N'douba : Son ... allô ... foo nekk?

Son-J : Eh fils !

N'douba : Maxim ñew na xoolal ... léegi mu tane xam nga ... ñu nekkalaat ko ci quoi xam nga ... comparaan nga ... waaw ... bii paa defa wara gën a mën a saf. Son-J : Mais franchement boy ... li ngay wax nii c'est bien beau mais j'ai envie de décrocher boy ... xam nga décrocher ... non fils franchement j'en ai ras-le-bol quoi.

N'douba : yaw lijjantiwu ñu loolu ... yaw bi nga nekkee fii yaw xam nga gang bi ni mu demee ... yaw ngay nekk fii xam nga ... di jéem a seral quoi !

Son-J : man instant yii nii dema bëgg samay moroom i gòor li ñu am man ma am ko quoi ... yaa ngi dégg ... dema bëgg am ci galle yu nice yi ... am ci trok yu nice yi quoi ... xam nga ... am jabar am ay doom xam nga ... gerer sama family nice ... c'est tout ce que je voudrais quoi.

N'douba : Fii rêve yooyu ñoo fi jeex fii gaalseen la ... man gëm u ma yooyu ... xoolal affaire bi defa war a mën a bombe ... mu tar ... xam nga ñu dund ko ñun.

Son-Ji : Gang bi ... man instant yii man dema ko bëgg a off.

N'douba: Non bul dekkal ... bayyil sa woo yi ... yaw xoolal ... mooy loolu.

Extrait 2 corpus 1 : Il s'agit d'un plateau de télévision où un journaliste animatrice de l'émission GUISS-GUISS ${ }^{17}$ discute avec ses invités sur le thème «Relation : cassure au moment le plus difficile de la vie ». L'émission est diffusée le 30 novembre 2018 à $8 \mathrm{~h}$ sur la chaine SENTV.

Invité : Su fekee jigèen defa nekk ci règle, yaayam ne ko sawal, mu saw si suuf normalement defa wara def bënn, nu xam ne kii, xam nga li may wax bu baax. Mais lèegi loolu yèpp de nu kaa dindi. Lèegi nun tamit de noo paré pour linjanti loolu xool li nu ci mën a reglé.

Animatrice: su ma deggèe li ngay wax nii defa mel ni parent yi de noo demissioné, d'accord. Mais rupture bi entre xale yi ak parent yi de nu koy noté tout le temps ndax juboo tu nu pask mag ñi buñ waxe xale yi duñ leen dèglu

Invité : Loolu yèpp education la ; bu nu jappoon education bi nu daan yoree seen doom yi ba lèegi mais parent yi lèegi lijantee tu nu seen doom telephone bi mu am de ngay gis sa doom yaw parent bi mo lay gën a seerle telephone, façon cheveux bu nekk am na ko te yaw doo lijanti sa doom fan la ko amee.

Extrait corpus 2 : les hybrides à l'université

Extrait 1, corpus 2. Il s'agit d'une discussion entre étudiant autour des « insulteurs sur le net ».

\footnotetext{
${ }^{16}$ Ibrahima Sarr ; «Le cinéma sénégalais : un cadre d'expression d'un parler jeune de ville ; Etude de la communication transcodique dans Tundu Wundu, un feuilleton de Abdoulabd Wone» dans Nodus Sciendi num 14, Septembre 2015.

${ }^{17}$ Guiss-Guiss est un mot compose wolof qui veut dire «Point de vue »
} 
Orateur A : C'est l'éducation de base qui n'a plus de rigueur au Galsen, magum jëmm taxaw si télé bi di saaga te dara du ko cifekk. Looloo tax su may dègg ñuy naan xale yi deñu reew sans dire ku yar xale yi ça m'enerve. Voilà, éthique $a k$ déontologie defa baax ci lu ne.

Orateur B : Man un copin qui va enseigner def ma woo di ma laaj conseil ; ma ne ko boy, bul mos a doxaan sa élève, loolu rekk laalay dènk en tant que petit frère. Boy bi di ma ree ma xam ne je prêche dans le vent.

Orateur $\mathbf{C}$ : Bu leen yaqq xew mi. Des adultes peuvent faire la différence sans grande difficulté. Dans les bureaux jëlante rekk lañuy def; pourquoi pas dans une classe ?

Orateur D : Dans d'autres systèmes the lecturer is not the one who gives marks ; $\mathrm{y}$ a des systèmes «fair» de QCM par exemple.

\section{Analyse des données}

Comme nous pouvons déjà le voir, l'hybridation présentée ici couvre principalement deux formes : il s'agit dans le premier cas de l'hybridation comme langue mixte, c'est-à-dire un parler qui emprunte les traits de plusieurs langues. Il s'agit clairement de pratiques transcodiques du genre mélange de codes et alternance de codes. Cette situation corrobore les propos de Moussa Daff (1998) qui affirme que la majeure partie des locuteurs wolof arrive difficilement à soutenir une conversation en langue wolof sans dévier de la norme ou se résoudre au transcodisme.

\section{Le transcodisme : cadre d'un partenariat linguistique}

Dans l'extrait 2 du corpus1 1'hybridation procède du mélange du wolof et du français. Bien que la langue de l'émission GUISS-GUISS soit le wolof, on note des mélanges par l'incorporation dans le discours de mots français, parfois adapté phonologiquement. Dans cette pratique, l'animatrice semble s'y adonner le plus. Cela pourrait être lié à la nature du thème débattu qu'ils semblent inscrire dans le technolecte de l'éducation. Dans cet extrait, un seul hybride lexical est noté et concerne le mot « seerle » adapté du français « cher » adjoint au morphème adjectival wolof «-le ». Il est à noter aussi que le plateau de cette émission regroupe des invités instruits et d'autre qui ne le sont pas, sinon avec un niveau d'étude beaucoup moins avancé. Chez ces derniers, la pratique du transcodisme s'observe dans une moindre mesure. La mixité dans cet extrait implique deux langues majoritaires de par leur statut et leurs fonctions dans la communication; il s'agit du Wolof et du français, respectivement langue nationale et langue officielle.

La deuxième forme d'hybridation apparait comme une interférence négative. Il s'agit d'alternance d'incompétence qui s'explique par le fait que le locuteur est semilingue, c'est-à-dire qu'il n'a de maitrise parfaite d'aucune des langues de son répertoire linguistique. Ce type est plus caractéristique des 
productions des étudiants du campus qui se distinguent des autres composantes de cet espace anthropo-social par une façon de parler qui obéit à des mécanismes linguistiques qui, d'un point de vue normatif, sont erronés. En effet, ces étudiants ont pour a plupart le wolof comme langue de socialisation, le français comme langue de travail et la langue maternelle si le locuteur n'est pas wolophone. Chacune de ces langues opère dans un cadre communicationnel spécifique où le degré d'exposition du locuteur ne suffit pas pour une intériorisation parfaite des ressources linguistiques.

Tout cela pourrait trouver une explication dans le fait que les locuteurs enquêtés parlent des langues du cercle central et périphérique et ont construit un bilinguisme vertical par le billet de l'école qui consacre le rôle de médium d'instruction au français (langue super-centrale) alors que l'anglais (hyperlangue ${ }^{18}$ et d'autres comme l'espagnol et l'arabe sont offertes en LV2. La diglossie et l'exposition à des degrés variés aux différentes langues de leur environnement expliquent ainsi une tendance à l'hybridation.

\section{Des positionnements épilinguistiques favorables a l'hybridation}

En principe, on peut penser que dans un contexte où l'Etat consent des efforts considérables pour l'émergence des parlures nationales et où les populations s'engagent dans la lutte contre la glottophagie, les attitudes linguistiques seront défavorables aux pratiques mixtes et que la conservation de la «pureté » des langues nationales demeure un défi. Cependant, les positionnements épilinguistiques exprimés sont tout autre avec une forte conscience à la nécessité de la protection de la diversité linguistique et du dialogue interculturel dont le transcodisme est considéré comme un mode d'expression. Par exemple, à la question « quel sentiment avez-vous vis-à-vis de la mixité dans l'expression des étudiants du campus ? "l'enquêté K.D répond : «Une bonne cohabitation, harmonie, mélange des cultures, c'est ça la vie ; c'est ça la société et je trouve que le multilinguisme nous rend plus solidaires. On partage et on apprend beaucoup de choses avec la multiplicité des langues et c'est très important; donc c'est un signe de paix et d'harmonie ». La même question posée à un journaliste dans le contexte des media reçoit une réponse similaire « vous savez, ce mélange de langues, c'est ce qui caractérise notre identité en tant que Sénégalais. Peut-être qu'on peut s'efforcer de ne pas s'y résoudre dans certains programmes comme le journal en français mais pour les autres émissions on est obligé, c'est normal ? » Ces positions offrent un reflet de l'attitude de la majorité et traduit des positionnements épilinguistiques nettement favorables aux pratiques hybrides. C'est également une telle posture qui a conduit Cheikh Tidiane Thiam dans

${ }^{18}$ Cf. Modèle gravitationnel de Louis-Jean Calvet. 
les termes de référence de son alphabet $\mathrm{ASA}^{19}$ à déclarer que l'identité Africaine est une mixité des apports de la civilisation arabo-musulmane, de la civilisation occidentale, moulées avec celle négro-africaine. La récurrence de cette pratique linguistique n'est pas non plus le fait d'une élite car comme nous pouvons le voir dans l'extrait du téléfilm Tundu Wundu d'Abdoulaye Wone, l'hybridation est également l'œuvre de toutes les couches sociales quelque soient le niveau d'étude, le sexe, la catégorie professionnelle etc.

\section{Conclusion}

L'objectif de cet article était de montrer le processus d'hybridation dans lequel se trouvent les langues du Sénégal, d'analyser comment ces formes reflètent les identités des locuteurs afin d'aborder la question des représentations linguistiques autour de cette mixité. L'analyse du corpus collecté permet effectivement de soutenir que l'hybridation linguistique est une réalité observable dans les pratiques langagières au Sénégal. Elle se présente sous deux formes, à savoir l'alternance de codes et l'interférence linguistique. Ce processus semble découler de la contiguïté géographique et des représentations positives des locuteurs. Ainsi, même s'il faut éviter de restreindre le phénomène à un groupe social spécifique, on peut néanmoins noter qu'il est très présent dans les média et dans l'espace académique qui sont des lieux de contact. Son acceptation et son expansion montrent qu'il est devenu un facteur identitaire dans un contexte où l'Etat dans sa politique éducative $^{20}$ et les locuteurs à travers des mouvements associatifs cherchent à donner plus de poids aux langues nationales.

\section{References:}

1. Babault, Sophie. «Plurilinguisme et tensions identitaires chez les lycéens malgaches » In Gottopol, N² - Juillet 2003.

2. Calvet, Louis-Jean. «Identité et multiculturalisme » Trois espaces linguistiques face aux défis de la mondialisation. Paris, 20 - 21 mars 2001.

3. Calvet, Louis-Jean. «Identité et multiculturalisme » Trois espaces linguistiques face aux défis de la mondialisation. Paris, 20 - 21 mars 2001.

\footnotetext{
${ }^{19}$ Alphabet de Synthèse Africaine. C'est un alphabet innovant, construit avec les lettres consonnes de l'alphabet du français, les voyelles de l'arabe et des signes diacritiques pour affiner les réalisations phonologiques.

${ }^{20}$ L'Etat du Sénégal a procédé à la mise à l'échelle de l'enseignement bilingue qui procède par l'intégration d'une langue nationale dans l'enseignement primaire. Les apprenants font ainsi leurs premiers pas à l'école dans une langue nationale et passe progressivement au français à mesure qu'ils avancent.
} 
4. Calvet, Louis-Jean. Les politiques linguistiques. Paris : Presses Universitaires de France. Coll. «Que sais-je ? »n³075, 1996.

5. Canut, Cécile. « À la frontière des langues. Figures de la démarcation » In Cahiers d'études africaines 2001/3-4, 163, p. 443-464.

6. CISSE, Mamadou. «Langues, Etat et société au Sénégal », in 'Sudlangue', n5. 2005, pp. 99-133 - accessible à http://www.ausenegal.com/IMG/pdf/doc-109pdf-33f96.pdf

7. Calvet, Louis-Jean. «La diversité linguistique : enjeux pour la Francophonie », Hermès, La Revue, vol. 40, no. 3, 2004, pp. 287-293.

8. Daff, Moussa. «L'aménagement linguistique et didactique de la coexistence du français et des langues nationales au Sénégal » dans DiversCité Langues. En ligne. Vol. III. 1998. Disponible à http://www.uquebec.ca/diverscite

9. Gadet, François. La variation sociale en français. Paris: Ophrys, 2003.

10. Juillard, Caroline. Sociolinguistique urbaine : la vie des langues à Ziguinchor. Paris : CNRS, 1995.

11. Ka, Fary Silate. «Politique linguistique du Sénégal des années 1960 à nos jours : réalités et perspectives » in Politiques linguistiques en Afrique francophone de l'indépendance à nos jours : bilan et perspectives. Kinshasa : 14-16 juin 2010. Accessible à http://faryka.emonsite.com/pages/communications/politiquelinguistique-du-senegal-1960-a-nos jours.html

12. Kerry Mullan, « Henri Boyer (éd.), Hybrides linguistiques. », Cahiers de praxématique [En ligne], 54-55 | 2010, document 22, mis en ligne le 01 janvier 2013, consulté le 02 octobre 2016. URL : http://praxematique.revues.org/1195.

13. Kortas, Jan. «Les hybrides lexicaux en français contemporain: délimitation du concept » dans Meta. Volume 54, Numéro 3, septembre, 2009, p. 533-550.

14. Labov, William. « Social and language boundaries among adolescents ». In American Speech, vol. 67, n4, 1996, p.339-366.

15. Mbaya, Maweja. Pratiques et attitudes linguistiques dans l'Afrique d'aujourd'hui : le cas du Sénégal.

16. Ndao P. A. \& Kébé A. B., 2010, «Langues et médias au Sénégal : une expérience de normalisation langagière par les journalistes des radios privées. Enjeux et limites », in Ndao P. A. \& Kébé A. B., (dirs.) Nouveaux médias et dynamiques des langues dans l'espace francophone, Glottopol $\mathrm{n}^{\circ} 14$, en ligne sur http://www.univrouen.fr/dyalang/glottopol/, pp. 17-36, [20.12.2017].

17. NDAO, Papa Alioune. «Politiques linguistiques et gestion de la diversité linguistique au Sénégal : aspects sociolinguistiques », in 
Glottopol $\quad n^{\circ} 18$. juillet 2011, pp.7-20.http://www.univrouen.fr/dyalang/glottopol. [1. 09. 2015]

18. Ndecky, Albinou. «Du pluralisme linguistique au fantasme du UN » In Langues et Litteratures. UGB, GELL, 2014

19. Sarr, Ibrahima. "The Issue of Identity in the Developmental Efforts of Senegalese Local Languages from 2001 Onwards » In International Journal of linguistics and communication, vol3, $n^{\circ} 2$. December 2015, pp. $1-5$

20. Sarr, Ibrahima. «Language Imperialism and the Fate of Minority Languages: Indoctrination through the So-Called Wolofisation of the Senegalese Society » In Journal of Foreign Languages, Cultures and Civilizations Vol. 2, No. 1, June 2014, pp. 93-104.

21. SENTV. «Relation : cassure au moment le plus difficile de la vie ». Emission GUISS-GUISS diffusée le 30 novembre 2018 à $8 \mathrm{~h}$.

Annexes : traduction française des extraits du corpus analyses (les séquences traduites sont en italiques, le reste est en langue d'origine)

\section{Extrait 1 corpus 1}

Son-J : Allô !

N'douba : Son ... allô ... tu es ou?

Son-J : Eh fils !

N'douba : Maxim est venu, tu sais ... il ira bientôt mieux... on pourra alors le réintégrer ... tu comprends ... ok ... cette fois on devra être plus sérieux.

Son-J : Mais franchement mon pote ... ce que tu dis là c'est bien beau mais j'ai envie de décrocher mon pote ... tu sais, décrocher ... non fils franchement j'en ai ras-le-bol quoi.

N'douba : Nous on se fou de ça ... quand tu es rentre dans le gang tu savais bien quelles étaient les règles ... et maintenant tu es la ... tu penses pouvoir tout lâcher quoi!

Son-J : moi, ce que je veux en ce moment c'est de réaliser ce que les hommes de mon âge ont su réaliser quoi ... tu vois ... je veux avoir une belle maison ... une belle bagnole ... tu comprends ... me marier, avoir des enfants, tu comprends... bien prendre soin de ma famille ... c'est tout ce que je voudrais quoi.

N'douba : Arrête de rêver, et reviens sur terre on est au Sénégal ... moi je ne crois pas à ces choses-là... écoute! L'affaire doit être plus sérieuse, plus ambitieuse, .c'est ça qu'il faut.

Son-Ji : Le Gang ... moi, à cette instant j'ai envie de le quitter.

N'douba: Non ne sois peureux... comporte-toi comme un homme ... écoute ... laisse tomber. 


\section{Extrait 2 corpus 1}

Invité : Lorsqu'une femme voit ses règles, normalement si sa maman lui demande de pisser au sol, la pie doit creuser un sillon et on saura que ... tu vois bien ce que je veux dire! Mais de nos jours tout cela n'existe plus. Et nous, nous sommes prêts pour considérer cette question et voir ce que nous pouvons y faire.

Animatrice : Si je comprends bien ce que tu dis c'est que les parents ont démissionné ! D’accord. Mais la rupture entre les enfants et les parent a été notée tout le temps car ils ne s'entendent plus parce que les enfants n'écoutent plus les ainés.

Invité : Tout cela est un problème d'éducation ; il fallait perpétuer éducation traditionnelle mais les parents ne se préoccupent plus de leurs enfants. Parfois en tant que parent tu remarques que ta fille a un téléphone beaucoup plus couteux que le tien, elle a tous les types de perruques mais tu ne te soucies même pas d'où elle a pu les avoir.

\section{Extrait 1, corpus 2}

Orateur A : C'est l'éducation de base qui n'a plus de rigueur au Sénégal. Il adulte se tient devant la télé et profêre des insultes dans l'impunité totale. C'est pour cela que lorsque j'entends dire que les enfants sont impolis sans indexer les éducateurs, ça m'énerve. Voilà, à toute chose il faut de l'éthique et de la déontologie.

Orateur B : Moi, un copain qui va enseigner $m$ 'a appelé pour me demander des conseils ; je lui ai dit, mon pote, abstiens-toi de toute relation amoureuse avec tes élèves, c'est tout ce que je te conseille en tant que petit frère. Le garçon s'est mis à se moquer de moi et j'ai compris que je prêche dans le vide. Orateur C: Il ne faut pas biaiser le débat. Des adultes peuvent faire la différence sans grande difficulté. Dans les bureaux ils se tissent des relations amoureuses; pourquoi pas dans une classe ?

Orateur D : Dans d'autres systèmes ce n'est pas l'enseignant qui donne les points; y a des systèmes «juste »; des QCM par exemple. 